\title{
MOBILIDADE DO PACLOBUTRAZOL EM UM SOLO FRANCO- ARENOSO CULTIVADO COM MANGA NO NORDESTE BRASILEIRO $^{(1)}$
}

\author{
Maria Aparecida Costa ${ }^{(2)}$, Valdemar Luiz Tornisielo ${ }^{(3)} \&$ Jussara \\ Borges Regitano $^{(4)}$
}

\begin{abstract}
RESUMO
No Brasil, os produtos de manga fazem uso intenso do paclobutrazol como regulador de crescimento. No entanto, pouco se sabe sobre seu potencial de transporte em condições de solo e clima brasileiros. O presente estudo teve como objetivo avaliar a sorção e a mobilidade do paclobutrazol (PBZ), um fitorregulador de crescimento do grupo químico triazol, em um solo franco-arenoso da região submédia no vale do rio São Francisco, onde é intenso o cultivo de manga. O estudo de sorção foi realizado por meio de isotermas, em cinco concentrações $(1,24 ; 2,48$; 5,$\left.08 ; 10,22 ; \mathrm{e} 20,52 \mathrm{mg} \mathrm{L}^{-1}\right)$, utilizando o produto radioativo ( $\left.{ }^{14} \mathrm{C}-\mathrm{PBZ}\right)$ como traçador. A dessorção foi avaliada após o descarte do sobrenadante e subseqüente adição de solução de $\mathrm{CaCl}_{2} \mathbf{0 , 0 1} \mathrm{mol} \mathrm{L}^{-1}$, etapa esta que foi repetida quatro vezes. Já a mobilidade do PBZ foi avaliada em colunas empacotadas com solo, com a simulação de $300 \mathrm{~mm}$ de chuva, uniformemente distribuída durante $72 \mathrm{~h}$. O PBZ apresentou baixo potencial de sorção no solo estudado $\left(\mathrm{K}_{\mathrm{f}}=1,06 \mathrm{mg}^{1-\mathrm{N}} \mathrm{L}^{\mathrm{N}} \mathrm{kg}^{-1} ; \mathrm{K}_{\mathrm{d}}(\right.$ médio $)=$ $0,83 \mathrm{~L} \mathrm{~kg}^{-1} ; \mathrm{e} \mathrm{K}_{\mathrm{OC}}$ (médio) $=165,7 \mathrm{~L} \mathrm{~kg}^{-1}$ ), o que correspondeu à sorção, em média, de $24,7 \%$ da quantidade aplicada. No processo de dessorção, apenas 5,3 \% do PBZ aplicado ficou retido às partículas do solo, $\mathrm{e} 19,4$ \% foi dessorvido à solução do solo. Portanto, em média, 75,3 \% do PBZ aplicado não foi sorvido e 19,4 \% foi dessorvido, totalizando 94,7 \% do produto disponível na solução do solo. Apesar disso, apenas $0,83 \%$ do PBZ aplicado foi lixiviado da coluna de solo, e $43,7 \%$ da quantidade aplicada foi transportada para além dos primeiros $10 \mathrm{~cm}$ de profundidade do solo. Portanto, a mobilidade do PBZ foi menor do que aquela esperada, por se tratar de um solo com elevado teor de areia $(77,5 \%)$ e baixo teor de C orgânico $\left(5 \mathrm{~g} \mathrm{dm}^{-3}\right)$ e em razão da grande quantidade de chuva simulada $(300 \mathrm{~mm})$. Mesmo assim, não
\end{abstract}

\footnotetext{
(1) Recebido para publicação em maio de 2007 e aprovado em setembro de 2008.

(2) Aluna de Pós-Doutorado, Laboratório de Ecotoxicologia, Centro de Energia Nuclear na Agricultura, Universidade de São Paulo - USP. Caixa Postal 97, CEP 13400-970 São Paulo (SP). Bolsista CNPq. E-mail:macosta@cena.usp.br

(3) Professor Doutor, Laboratório de Ecotoxicologia, Centro de Energia Nuclear na Agricultura, USP. E-mail: vltornis@cena.usp.br

(4) Pesquisadora, Laboratório de Ecotoxicologia, Centro de Energia Nuclear na Agricultura, USP. E-mail: regitano@cena.usp.br
} 
se pode descartar, pelo menos totalmente, a possibilidade de que possa ocorrer lixiviação do PBZ, uma vez que ele mostrou mobilidade no perfil da coluna.

Termos de indexação: sorção, lixiviação, coluna de solo, regulador de crescimento, triazol.

\title{
SUMMARY: MOBILITY OF PACLOBUTRAZOL IN AN INTENSIVELY USED SANDYLOAMSOIL UNDERMANGOINNORTHEASTERNBRAZIL
}

\begin{abstract}
In Mango cultivation under Brazil conditions paclobutrazol (PBZ), a growth regulator, is very used. However it is not yet well known about the leaching potential of PBZ in these soil conditions. The objectives of this study were to evaluate sorption and mobility of paclobutrazol (PBZ), a growth regulator of the chemical group triazol, in a sandy loam soil found in the sub-mid region of the San Francisco River valley, where mango cultivation is intense. The sorption study was based on isotherms, at five different concentrations (1.24; 2.48; 5.08; 10.22; and $\left.20.52 \mathrm{mg} \mathrm{L}^{-1}\right)$, using the radioisotope $\left({ }^{14} \mathrm{C}\right.$-PBZ) as tracer. Desorption was evaluated after discarding the supernatant and further addition of $0.01 \mathrm{~mol} \mathrm{~L}^{-1} \mathrm{CaCl}_{2}$ solution, and repeating the entire process four times. The PBZ mobility was evaluated in packed soil columns, under an evenly distributed rainfall simulation of $300 \mathrm{~mm}$ for $72 \mathrm{~h}$. The PBZ detected low sorption potential in the studied soil $\left(K_{f}=1.06 \mathrm{mg}^{1-N} \mathrm{~L}^{N} \mathrm{~kg}^{-1}\right.$; $K_{d}$ $($ mean $)=0.83 \mathrm{~L} \mathrm{~kg}^{-1}$ and $K_{O C}($ mean $\left.)=165.7 \mathrm{~L} \mathrm{~kg}^{-1}\right)$, corresponding to a mean of $24.7 \%$ of the applied amount. After desorption, only $5.3 \%$ of the applied PBZ was still sorbed to the soil particles, thus $19.4 \%$ of the applied amount was desorbed to the soil solution. Therefore, $75.3 \%$ of the applied PBZ remained in solution after sorption and $19.4 \%$ was desorbed, totalizing an amount of $94.7 \%$ of the compound available in the soil solution. However, only $0.83 \%$ of the applied PBZ leached through the soil columns, but $43.7 \%$ of the applied amount was transported below the upper $10 \mathrm{~cm}$ of the soil profile. PBZ mobility was therefore lower than expected, even when taking the high sand (77.5\%) and low organic $C$ $\left(5 \mathrm{~g} \mathrm{dm}^{-3}\right)$ soil contents into consideration and that rainfall simulation was rather intense $(300 \mathrm{~mm})$. Nevertheless, the possibility of leaching could not be ruled out in view of the PBZ mobility in the soil profile.
\end{abstract}

Index terms: sorption, leaching, soil columns, growth regulator, triazol.

\section{INTRODUÇÃO}

No Brasil, a fruticultura ocupa cerca de $5 \%$ da área cultivada e representa uma atividade capaz de assegurar ao País, segundo o Ministério da Agricultura, Pecuária e Abastecimento (MAPA), o primeiro lugar mundial entre os produtores de frutas in natura, podendo aumentar o poder aquisitivo da classe média na região Nordeste. Por exemplo, a região do semi-árido brasileiro apresenta agricultura irrigada intensiva de fruteiras, cujo retorno social é expressivo para a própria fixação do homem no campo.

No submédio do rio São Francisco, a produção de mangas é explorada por pequenos e grandes produtores, que contribuem com cifras expressivas para a economia nacional mediante a exportação desses produtos. No entanto, o cultivo irrigado intensivo em associação às condições climáticas locais e ao tipo de solo - na maioria arenosos - favorece o aparecimento de pragas e doenças, o que requer a utilização intensiva de pesticidas e outros insumos para o aumento ou, até mesmo, a manutenção da produtividade (Ferracini et al., 2001). No entanto, a elevada utilização desses insumos pode contaminar e, portanto, comprometer a qualidade do solo e das águas superficiais e subterrâneas, ameaçando a preservação do meio ambiente. O problema se torna ainda mais crítico quando se considera a grande demanda de água para o consumo humano e a irrigação de frutíferas na região.

O solo, normalmente, é o último reservatório natural para os pesticidas e outros insumos agrícolas. A partir desse compartimento, esses produtos podem ser liberados para a atmosfera, para as fontes de águas e para os organismos vivos - principalmente para os microrganismos. Os pesticidas, assim como os outros insumos, podem ainda permanecer ativos no solo por longos períodos, interferindo diretamente, ou mesmo impedindo, no desenvolvimento de novas culturas (Luchini, 1987).

Quando atingem o solo, os pesticidas e os insumos agrícolas podem ficar retidos junto à superfície das partículas de solo, pelo processo conhecido como sorção. 
A reação de sorção, de forma geral, limita o transporte desses produtos para outros compartimentos ambientais, pois limita sua disponibilidade na solução do solo. Convém salientar que o equilíbrio da reação de sorção é um processo reversível, ou melhor, parcialmente reversível e dinâmico; portanto, pode ocorrer a liberação dos pesticidas e insumos para a solução do solo por meio da dessorção. No entanto, a cinética da dessorção costuma ser muito mais lenta que a da reação de sorção.

Dessa forma, fica claro que o processo de sorção é fundamental para tentar predizer o destino de um insumo agrícola no ambiente, pois essa reação está diretamente relacionada à disponibilidade desses produtos no ambiente, alterando sua translocação, persistência, mobilidade e bioatividade no solo e nas plantas presentes (Luchini, 1987). Além da sorção, existem outros testes específicos para avaliar a mobilidade de um produto no solo. Normalmente, a mobilidade é avaliada usando colunas de solo empacotadas (OECD, 2004). Apesar das limitações deste tipo de estudo (uso de amostras de solo deformadas, dimensão da coluna $-5 \mathrm{~cm}$ de diâmetro e $30 \mathrm{~cm}$ de profundidade, fluxo contínuo e distribuição uniforme de chuva), ele oferece informações mais conclusivas quanto ao potencial de mobilidade de uma molécula do que o teste de sorção, uma vez que o produto tem de atravessar um "volume" de solo com uma quantidade razoável de água a ser percolada.

Os produtores de manga utilizam o paclobutrazol (PBZ), também conhecido como Cultar, para controle do crescimento, redução da poda e manipulação do cultivo para expansão da produção. Segundo Davis \& Curry (1991), o PBZ atua por meio da inibição da síntese das giberelinas; a forma ativa do composto alcança os meristemas subapicais e inibe a oxidação do caureno para ácido caurenóico, que é o precursor do ácido giberélico. Essa reação é catalisada pelo citocromo P-450 e ocorre nos microssomos, resultando em redução da divisão celular, sem causar fitotoxicidade. Como conseqüência morfológica direta, ocorre redução do vigor vegetativo, o que restringe o crescimento da planta e possibilita melhor manipulação e manejo da cultura (Davis \& Curry, 1991).

$\mathrm{Na}$ literatura encontram-se poucas informações sobre o destino do PBZ no ambiente. Jackson et al. (1996) observaram degradação microbiana parcial do PBZ pela ação das bactérias Pseudomonas e Alcaligenes, com o anel 1,2,4-triazol mostrando-se resistente ao ataque microbiano. Silva et al. (2003) verificaram que os solos que já haviam recebido aplicação de PBZ apresentaram maior taxa de biodegradação do produto. Nesses solos, provavelmente, os microrganismos estariam mais adaptados à utilização do PBZ como fonte de C. No entanto, mesmo após a inoculação dos solos com bactérias altamente eficientes na degradação do PBZ, observou-se que sua degradação foi lenta, apresentando meia-vida $\left(\mathrm{DT}_{50}\right)$ de 95 dias.
De acordo com os critérios adotados pela EPA (Cohen et al., 1995) e pelo modelo Groundwater Ubiquity Score (Gustafson, 1989), os quais se baseiam em algumas propriedades físico-químicas do produto (no caso do PBZ, têm-se: solubilidade em água = $35 \mathrm{mg} \mathrm{L}-1, \mathrm{~K}_{\mathrm{OC}}=66 \mathrm{~L} \mathrm{~kg}^{-1}, \mathrm{DT}_{50}=180$ dias e $\mathrm{K}_{\mathrm{H}}=$ $3,9 \times 10^{-2} \mathrm{~Pa} \mathrm{~m}^{3} \mathrm{~mol}^{-1}$ ), pode-se concluir que o $\mathrm{PBZ}$ apresenta alto potencial de mobilidade para fontes de água subterrâneas (Ferracini et al., 2001). Por outro lado, Leonard (1986) relatou que o perigo de contaminação de águas pelo processo de lixiviação é bastante reduzido com o PBZ, uma vez que ele apresenta baixo potencial de mobilidade no solo (Classificação de Helling: 1-2). No entanto, esse relato deve ser analisado com cautela, pois o valor do coeficiente de sorção $\left(K_{d}\right)$ do PBZ pode variar consideravelmente com o tipo de solo, podendo apresentar valores que variam de 1,5 L kg-1, em solos arenosos, a até $22,5 \mathrm{~L} \mathrm{~kg}^{-1}$, em solos argilosos com maiores teores de matéria orgânica. Sabe-se que o PBZ apresenta alta afinidade pela fração orgânica do solo. Esse resultado enfatiza que o PBZ pode apresentar tanto baixo como alto potencial de mobilidade, dependendo do tipo de solo e das condições climáticas.

Pouco é conhecido sobre a mobilidade do PBZ em condições tropicais, principalmente em solo e clima brasileiros. Assim, é necessário desenvolver estudos que diagnostiquem o impacto resultante de sua aplicação em nossas condições ambientais. Portanto, nesta pesquisa avaliaram-se os potenciais de sorção e mobilidade do PBZ em um solo franco-arenoso, muito utilizado para cultivo da manga da região submédia no vale do rio São Francisco.

\section{MATERIAL E MÉTODOS}

\section{Solo}

Amostras da camada superficial (0 a 0,2 m) de um solo franco-arenoso, intensivamente cultivado com manga, foram coletadas na região submédia no vale do rio São Francisco, em Petrolina. O solo apresentou as seguintes propriedades físico-químicas: $35,8 \%$ de areia grossa; 41,7 \% de areia fina; $8,3 \%$ de silte; $14,2 \%$ de argila; $5 \mathrm{~g} \mathrm{dm}^{-3}$ de matéria orgânica; $\mathrm{pH}-\mathrm{CaCl}_{2}$ de 5,4 ; e capacidade de troca catiônica $(\mathrm{T})$ de 19,4 $\mathrm{mmol}_{\mathrm{c}} \mathrm{dm}^{-3}$. Após a coleta, as amostras de solo foram secas ao ar e passadas em peneira de $2 \mathrm{~mm}$.

\section{Produto químico}

O paclobutrazol, ([2RS, 3RS]-1-[4-clorofenil]-4,4dimetil-2-[1,2,4-triazol-1-il]-pentano-3-ol), pertencente à classe fitorregulador do grupo químico triazol, com classificação toxicológica classe III, foi testado neste experimento (Figura 1). Para os testes, tanto o produto técnico como o isótopo radioativo (posição de marcação $={ }^{14} \mathrm{C}$-triazol, atividade específica $=3,58 \mathrm{mCi} \mathrm{g}^{-1} \mathrm{e}$ pureza radioquímica $\geq 95 \%$ ) foram utilizados. 
<smiles>CC(C)C(O)C(Cc1ccc(Cl)cc1)n1cncn1</smiles>

Figura 1. Estrutura química do paclobutrazol e localização da marcação com ${ }^{14} \mathrm{C}(*)$.

\section{Isotermas de sorção e dessorção}

Para o procedimento do estudo de isotermas de sorção, foram preparadas cinco soluções de PBZ, nas seguintes concentrações: 1,$24 ; 2,48 ; 5,08 ; 10,22$; e $20,52 \mathrm{mg} \mathrm{L}^{-1}$, em $\mathrm{CaCl}_{2} 0,01 \mathrm{~mol} \mathrm{~L}^{-1}$ e concentração radioativa de aproximadamente $0,16 \mathrm{kBq} \mathrm{mL}^{-1}$. Alíquotas de 5,0 $\mathrm{mL}$ dessas soluções, em triplicata, foram adicionadas a amostras de $2 \mathrm{~g}$ de solo (peso do solo seco em estufa), devidamente acondicionadas em tubos de centrífuga, os quais foram agitados horizontalmente a 120 revoluções por minuto, durante $16 \mathrm{~h}$. Amostras em branco (solução sem o solo) foram agitadas em paralelo, para determinar a concentração inicial do produto em solução. Após o período de agitação, os tubos foram centrifugados a $12.100 \mathrm{~g}$, durante $15 \mathrm{~min}$; alíquotas de $1,0 \mathrm{~mL}$ da solução sobrenadante foram retiradas e adicionadas a uma solução cintiladora para determinação da concentração de equilíbrio $\left(\mathrm{C}_{e}\right)$ do $\mathrm{PBZ}$, por espectrometria de cintilação líquida (ECL). A concentração sorvida (S) de PBZ foi determinada pela diferença entre a concentração inicial $\left(\mathrm{C}_{j}\right)$ e a concentração de equilíbrio $\left(\mathrm{C}_{e}\right)$ do $\mathrm{PBZ}$, ou seja, $\mathrm{S}=\left(\mathrm{C}_{i}-\mathrm{C}_{e}\right) \times(\mathrm{V} / \mathrm{M})$, em que $\mathrm{V}=$ volume da solução e $\mathrm{M}=$ massa de solo.

Os resultados foram ajustados à equação linearizada de Freundlich: $\log \mathrm{S}=\log \mathrm{K}_{\mathrm{f}}+\mathrm{N} \log \mathrm{C}_{e}$, em que $\mathrm{K}_{\mathrm{f}}=$ constante de sorção de Freundlich e N = coeficiente exponencial da curva. O coeficiente de sorção linear $\left(\mathrm{K}_{\mathrm{d}}\right.$, assumindo-se $\left.\mathrm{N}=1\right)$ foi calculado como a média dos valores obtidos para cada concentração, a partir do qual se pode calcular o valor do coeficiente de sorção normalizado ao teor de $\mathrm{C}$ orgânico do solo $\left(\mathrm{K}_{\mathrm{oc}}=\mathrm{K}_{\mathrm{d}} / \mathrm{C}_{\text {org }}\right)$.

Para o estudo de dessorção, os sobrenadantes foram descartados e alíquotas de $5 \mathrm{~mL}$ da solução de $\mathrm{CaCl}_{2}$ $0,01 \mathrm{~mol} \mathrm{~L}^{-1}$ foram adicionadas aos tubos de centrífuga, sendo anotados os valores dos pesos para o cálculo da solução remanescente. Novamente, os tubos foram agitados, centrifugados, e alíquotas de 1,0 mL da solução sobrenadante removidas para determinação da fração dessorvida do PBZ. A radioatividade foi determinada por ECL, sendo esse procedimento repetido por mais três vezes. Isso permitiu calcular a percentagem de PBZ dessorvida.

\section{Lixiviação}

Neste experimento foram utilizadas colunas de vidro ( $5 \mathrm{~cm}$ de diâmetro e $40 \mathrm{~cm}$ de comprimento), com extremidade afunilada nos últimos $5 \mathrm{~cm}$. Para o preenchimento das colunas, foi colocada uma camada de lã de vidro seguida de areia esterilizada, até preencher a parte afunilada da coluna. Em seguida, as colunas foram preenchidas com solo até $28 \mathrm{~cm}$ de altura, sendo compactada por trepidação com o auxílio de um agitador de tubos. No topo também foi colocada lã de vidro, para promover distribuição uniforme da água durante o gotejamento. A densidade e a porosidade do solo empacotado foram calculadas com dados do volume da coluna (densidade de empacotamento $=1,73 \mathrm{~g} \mathrm{~cm}^{-3}$ e porosidade $=34,5 \%$ ).

As colunas de solo (em triplicatas) foram saturadas com água destilada, por capilaridade, e o excesso de água foi percolado por $2 \mathrm{~h}$. Então, elas foram acondicionadas em sala escura, climatizada $\left(25 \pm 2{ }^{\circ} \mathrm{C}\right)$, e o PBZ foi aplicado na dose de $1.000 \mathrm{~g} \mathrm{ha}^{-1}$ i.a. (ou 196,36 $\mu \mathrm{g}$ de PBZ/coluna), com concentração radioativa de $25,99 \mathrm{kBq} /$ coluna. Posteriormente, chuva foi simulada à taxa constante de $0,14 \mathrm{~mL} \mathrm{~min}^{-1}$, com o auxílio de uma bomba peristáltica, totalizando $300 \mathrm{~mm}$ de chuva em $72 \mathrm{~h}$. O lixiviado foi coletado em intervalos de $12 \mathrm{~h}$, cujo volume foi medido por gravimetria e a radioatividade determinada por ECL.

Após $72 \mathrm{~h}$, as colunas foram armazenadas em freezer $\left(-16{ }^{\circ} \mathrm{C}\right.$, por $\left.24 \mathrm{~h}\right)$, para facilitar a secção das camadas a cada $5 \mathrm{~cm}$ de solo, as quais foram secas ao ar e pesadas. Para se determinar a radioatividade das diferentes camadas de solo, subamostras de $0,4 \mathrm{~g}$, em triplicatas, foram queimadas em oxidador biológico (900 ${ }^{\circ} \mathrm{C}$, por $3 \mathrm{~min}$ ). $\mathrm{O}^{14} \mathrm{CO}_{2}$ desprendido foi capturado pela monoetanolamina, sendo a radioatividade determinada por ECL.

\section{RESULTADOS}

Para todos os estudos aqui realizados, os valores dos coeficientes de variação entre as repetições foram inferiores a $10 \%$.

\section{Sorção/dessorção}

Para o solo estudado, observou-se que $\mathrm{K}_{\mathrm{f}}$ foi igual a $1,06 \mathrm{mg}^{1-\mathrm{N}} \mathrm{L}^{\mathrm{N}} \mathrm{kg}^{-1}(\mathrm{~N}=0,80)$. Portanto, o potencial de sorção do PBZ foi baixo para o solo estudado, ou seja, a quantidade sorvida de PBZ diminuiu de 29,5 para $18,7 \%$ da quantidade aplicada com o aumento da concentração inicial da solução de 1,24 para 20,52 $\mathrm{mg} \mathrm{L}^{-1}$ de PBZ. Paralelamente, $\mathrm{K}_{\mathrm{d}}$ (médio) foi igual a $0,83 \mathrm{~L} \mathrm{~kg}^{-1}$, diminuindo de 1,05 para $0,58 \mathrm{~L} \mathrm{~kg}^{-1}$ com o aumento da concentração inicial da solução. Portanto, o valor de $\mathrm{K}_{\mathrm{oc}}$ variou entre $115,1 \mathrm{e}$ $209,6 \mathrm{~L} \mathrm{~kg}^{-1}$, com valor médio de $165,7 \pm 38,0 \mathrm{~L} \mathrm{~kg}^{-1}$ de $\mathrm{C}$ orgânico.

No estudo de dessorção, observou-se que, em média, apenas 5,3 \% da quantidade aplicada de PBZ permaneceu retida às partículas do solo; $19,4 \%$ da quantidade aplicada (ou 78,5 \% da quantidade sorvida) foi liberada no processo de dessorção; e 75,3 \% foi 
descartada junto com a solução sobrenadante, após o processo de sorção. Portanto, 94,7 \% (19,4 + 75,3) do PBZ aplicado ao solo mostrou-se disponível na solução do solo.

\section{Lixiviação}

A quantidade de PBZ lixiviada foi de apenas $0,83 \%$ do aplicado (Quadro 1).

Apesar disso, houve redistribuição do produto no perfil da coluna de solo, ou seja, aproximadamente 43,7 \% do produto aplicado foi transportado para além dos primeiros $10 \mathrm{~cm}$ de profundidade (Figura 2). Paralelamente, observou-se que 53,7\% do aplicado permaneceu na camada superficial (nos primeiros $10 \mathrm{~cm}$ da coluna). Neste experimento, 98,2\% da radioatividade aplicada foi recuperada.

Apesar do elevado teor de areia (77,5\%), do baixo teor de carbono orgânico $\left(5 \mathrm{~g} \mathrm{dm}^{-3}\right)$, do baixo potencial de sorção $\left(\mathrm{K}_{\mathrm{d}}=0,83 \mathrm{~L} \mathrm{~kg}^{-1}\right)$ e da quantidade de chuva (300 mm), a quantidade de PBZ lixiviada não foi tão acentuada. Esse resultado sugere que o potencial de

Quadro 1. Percentagem de paclobutrazol coletado no lixiviado, após intervalos de $12 \mathrm{~h}$. (chuva simulada de $300 \mathrm{~mm}$, uniformemente distribuída durante $72 \mathrm{~h}$ )

\begin{tabular}{cc}
\hline Período & Lixiviado \\
\hline $\mathrm{h}$ & \% de PBZ aplicado \\
12 & 0,02 \\
24 & 0,11 \\
36 & 0,24 \\
48 & 0,13 \\
56 & 0,21 \\
72 & 0,13 \\
Total & 0,83 \\
\hline
\end{tabular}

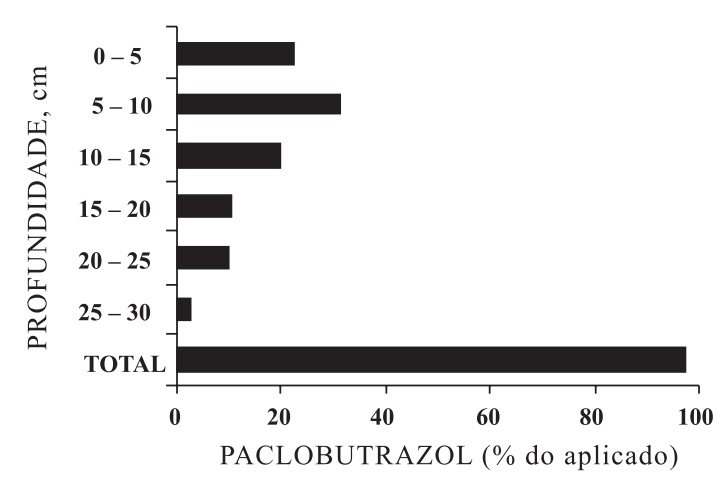

Figura 2. Mobilidade do paclobutrazol no perfil da coluna de solo (\% do aplicado). lixiviação do PBZ em condições reais de campo seja menor do que aquele previsto pelas características da molécula e da sua interação com o solo, como solubilidade, potencial de sorção, persistência (meiavida) e coeficiente de Henry $\left(\mathrm{K}_{\mathrm{H}}\right)$, conforme foi especulado por Ferracini et al. (2001).

Mesmo assim, por este estudo, não se pode descartar, pelo menos não totalmente, a possibilidade de que possam ocorrer problemas relacionados à lixiviação do PBZ, principalmente nesse tipo de solo, uma vez que esse produto apresentou mobilidade no perfil da coluna.

\section{CONCLUSÕES}

1. O potencial de sorção do PBZ foi baixo no solo estudado $\left(\mathrm{K}_{\mathrm{d}}=0,83 \mathrm{~L} \mathrm{~kg}^{-1}\right)$, mostrando potencial de mobilidade.

2. A quantidade lixiviada foi pequena $(0,83 \%) \mathrm{em}$ relação à aplicada, mas $43,7 \%$ do $\mathrm{PBZ}$ aplicado foi transportado para além dos primeiros $10 \mathrm{~cm}$ de profundidade do solo.

\section{LITERATURA CITADA}

COHEN, S.Z.; WAUCHOPER, R.D.; KLEIN, A.W.; EADSPORTH, C.V. \& GRANCY, R. Offsite transport of pesticides in water: Mathematical models of pesticide leaching and runoff. Pure Appl. Chem., 67:2109-2148, 1995.

DAVIS, T.D. \& CURRY, E.A. Chemical regulation of vegetative growth. Crit. Rev. Plant Sci., 10:151-188, 1991.

FERRACINI, V.L.; PESSOA, M.C.Y.P.; SILVA, A.S. \& SPADOTTO, C.A. Análise de risco de contaminação das águas subterrâneas e superficiais da região de Petrolina (PE) e Juazeiro (BA). Pesticidas: R. Ecotoxicol. Meio Amb., 11:1-16, 2001.

GUSTAFSON, D.I. Groundwater ubiquity score: A simple method for assessing pesticide leachability. Environ. Toxicol. Chem., 8:399-357, 1989.

JACKSON, M.J.; LINE, M.A. \& HASAN, O. Microbial degradation of a recalcitrant plant growth retardantpaclobutrazol (PP333). Soil Biol. Biochem., 28:1265-1267, 1996.

LEONARD, W.F. Cultar - A plant growth regulator for horticulture. New Zealand Agric. Sci., 20:195-202, 1986.

LUCHINI, L.C. Adsorção-dessorção dos herbicidas paraquat, diuron e 2, 4-D em seis solos brasileiros. Piracicaba, Escola Superior de Agricultura "Luiz de Queiroz", 1987. 91p. (Tese de Mestrado) 
ORGANIZATION FOR ECONOMIC CO-OPERATION AND DEVELOPMENT - OECD. Guidelines for the testing of chemicals. Leaching in soils columns. Paris, 2004. 15p. (Test 312)
SILVA, C.M.M.S.; FAY, E.F. \& VIEIRA, F.V. Degradação do paclobutrazol em solos tropicais. Pesq. Agropec. Bras., 38:1223-1227, 2003. 